\title{
FUS Oncofusion Protein Condensates Recruit mSWI/SNF Chromatin Remodelers via Heterotypic Interactions Between Prion-like Domains
}

Richoo B. Davis ${ }^{1}$, Taranpreet Kaur $^{1}$, Mahdi Muhammad Moosa ${ }^{1}$, Priya R. Banerjee ${ }^{1 *}$

${ }^{1}$ Department of Physics, University at Buffalo, Buffalo, NY, 14260, USA

* Correspondence should be addressed to P. R. B. email: prbanerj@buffalo.edu 


\begin{abstract}
Fusion transcription factors generated by genomic translocations are common drivers of several types of cancers including sarcomas and leukemias. Oncofusions of the FET (FUS, EWSR1, and TAF15) family of proteins result from fusion of the prion-like domain (PLD) of FET proteins to the DNA-binding domain (DBD) of certain transcription regulators and are implicated in aberrant transcriptional programs through interactions with chromatin remodelers. Here, we show that FUS-DDIT3, a FET oncofusion protein, undergoes PLD-mediated phase separation into liquid-like condensates. Nuclear FUS-DDIT3 condensates can recruit essential components of the global transcriptional machinery such as the chromatin remodeler SWI/SNF. The recruitment of mammalian SWI/SNF is driven by heterotypic PLD-PLD interactions between FUS-DDIT3 and core subunits of SWI/SNF, such as the catalytic component BRG1. Further experiments with single-molecule correlative force-fluorescence microscopy support a model wherein the fusion protein forms condensates on DNA surface and enrich BRG1 to activate transcription by ectopic chromatin remodeling. Similar PLD-driven co-condensation of $\mathrm{mSWI} / \mathrm{SNF}$ with transcription factors can be employed by other oncogenic fusion proteins with a generic $P L D-D B D$ domain architecture for global transcriptional reprogramming.
\end{abstract}




\section{Introduction}

FET fusion proteins are key drivers of several types of cancers including sarcomas and leukemias $[1,2]$. These chimeric proteins are created by the oncogenic fusion of two nonhomologous genes [3-5]. In the case of FET (FUS, EWSR1, and TAF15) family fusion proteins, their N-terminal disordered domain fuse to the DNA-binding domain of the transcription factor family ETS (E-twenty-six). The N-terminal domain of FET proteins features a low complexity sequence enriched in aromatic and polar amino acids $(\mathrm{Q} / \mathrm{N} / \mathrm{Y} / \mathrm{S} / \mathrm{G})$ and is classified as 'prionlike' [6]. Prion-like domains (PLDs) are present in nearly $1 \%$ of the human proteome, predominantly in ribonucleoproteins (RNPs) including the FET proteins, TDP43, TIA1, and hnRNPA1 [7, 8]. PLD-containing proteins are highly enriched in various biomolecular condensates such as stress granules and transcription factor condensates [7, 9-11]. At the molecular level, PLDs enable the formation of dynamic protein condensates through a physical process known as liquid-liquid phase separation (LLPS), which is mediated by multivalent selfinteractions among PLD chains involving distributed aromatic residues [12].

Although the phase separation of PLDs has been well characterized in the context of RNA binding proteins [13], the impact of PLDs fused to DNA-binding proteins such as transcription factors is relatively less explored. Transcription factors typically utilize their DNAbinding domain (DBD) to bind to specific gene loci and use their activation domains to recruit additional regulatory coactivators and RNA Polymerase II [14-17]. For FET oncofusion transcription factors, PLDs can act as the activation domain providing additional functionalities to the fusion transcription factors including the capacity to form phase-separated transcriptional hubs [18, 19]. Moreover, these fused PLDs may establish new interactions or modulate interactions with existing partners of the oncofusion transcription factor.

Recent studies have provided evidence that the PLDs of FET fusion oncoproteins interact with the mammalian SWI/SNF (mSWI/SNF) chromatin remodeling complex [2, 20-22]. The $\mathrm{mSWI} / \mathrm{SNF}$ or BAF is an evolutionarily conserved multiprotein complex that uses ATP hydrolysis to reposition nucleosomes and remodel chromatin landscape [23-25]. The mSWI/SNF represents a wide variety of complexes with varying subunit compositions that are expressed in a developmental stage and tissue-specific manner [26, 27]. Predominantly localized at enhancers and promoters, $\mathrm{mSWI} / \mathrm{SNF}$ plays key roles in regulating transcription [28]. Mutations in subunits of this complex are documented in $\sim 20 \%$ of all cancers implying a high propensity for oncogenesis in the event of their dysregulation and/ or mislocalization [20, 23, 29, 30]. Since the subunits of mSWI/SNF lack DNA-recognition motifs, their recruitment to specific genomic locations is typically mediated via their interactions with transcription factors and coactivators $[28,31,32]$.

In this study, we use the FET oncofusion FUS-DDIT3 as a model system to investigate the molecular behavior of such fusion oncoproteins and the mechanism of their engagement with the mSWI/SNF chromatin remodeler. FUS-DDIT3 (Type II fusion [33]; Fig. 1a) is composed of the PLD of FUS fused to the ETS family transcription factor, DDIT3 (see Table B: Materials \& Methods for protein sequence), and is detected in more than $90 \%$ of the myxoid/round cell 
liposarcoma [34, 35]. Our results suggest that the PLD drives robust phase separation of FUSDDIT3 in the mammalian cell nucleus, which is unlike the behavior of the parent protein FUS that remains predominantly soluble due to its interactions with nuclear RNAs. We further uncover the existence of PLDs in multiple subunits of the mSWI/SNF complex that can enable a synergistic engagement with the FET fusion oncoproteins through heterotypic PLD-PLD interactions. Such interactions provide a molecular mechanism for the recruitment of the $\mathrm{mSWI} / \mathrm{SNF}$ to FET-oncoprotein condensates and reveal a molecular pathway for rewiring transcriptional programs by aberrant chromatin remodeling.

\section{Results}

\section{FUS-DDIT3 undergoes LLPS in vitro and in mammalian cells}

Prior studies have established a key role of the prion-like domain (PLD) in driving the phase separation of FUS into liquid-like condensates [13, 36, 37]. We, therefore, wanted to test if the PLD enables FUS-DDIT3 fusion protein to undergo a similar liquid phase condensation. Utilizing recombinant FUS-DDIT3, we found that the fusion oncoprotein forms condensates in vitro in a concentration-dependent manner (Fig. 1b). FUS-DDIT3 condensates were stable across a broad range of $\mathrm{NaCl}$ concentrations (10-300 mM, Fig. S1a), and the presence of polymer crowders facilitated their formation (Fig. S1b). Fluorescence recovery after photobleaching (FRAP) experiments showed that FUS-DDIT3 molecules within these condensates are dynamic, implying a liquid-like behavior (Fig. 1c). Interestingly, we observed that FUS-DDIT3 assemblies appear as a mixture of "irregular" bodies and spherical condensates at lower protein and crowder concentrations but form predominantly large spherical condensates at higher concentrations $\left(\mathrm{C}_{\text {FUS-DDIT3 }}>50 \mu \mathrm{M}\right.$ and Ficoll PM70 $\geq 10 \%$; Figs.1b \& S1b). This is analogous to the previously reported study on SPOP-DAXX condensates where competition between inter-chain interactions of variable strengths determine the material state of the resultant assemblies [38, 39]. When compared with the PLD of FUS alone, we observed that the FUS-DDIT3 fusion protein formed condensates at a significantly lower protein concentration (Fig.1b \& S1c). These data suggest that DDIT3 may directly contribute to the homotypic interactions between FUS-DDIT3 molecules. Consistent with this idea, we observed that recombinant DDIT3 itself can form micron-scale assemblies in vitro that appear gel-like and are less dynamic than FUS-DDIT3 assemblies (Figs. S1d\&e). Thus, intermolecular PLD-PLD interactions along with DDIT3DDIT3 interactions synergistically facilitate the formation of FUS-DDIT3 condensates.

To investigate the phase behavior of FUS-DDIT3 in cell culture models, we expressed GFP-tagged FUS-DDIT3 in HEK293T cells and observed the formation of FUS-DDIT3 enriched spherical assemblies in the nucleus (Fig. 1d, center panel). These FUS-DDIT3 foci were dynamic as evidenced by their near-complete fluorescence recovery after photobleaching (Fig. 1e). We also observed that FUS-DDIT3 droplet/foci formation was concentration-dependent as cells with low concentrations of transgenically expressed FUS-DDIT3 lacked these foci (Fig. S1f), suggesting that the oncofusion protein's condensation is mediated via an LLPS mechanism. On the contrary, both GFP-DDIT3 and GFP-FUS showed a diffused distribution in the nucleus, with 
small punctate structures observed for GFP-FUS (Fig. 1d). Taken together, these results demonstrate that the PLD of FUS when fused to the transcription factor DDIT3 can drive phase separation of the fusion protein both in vitro and in the cell nucleus.

\section{FUS-DDIT3 forms stable condensates within the RNA-rich nuclear environment}

To understand the distinct behavior of FUS-DDIT3 and the parent FUS protein in the nucleus, we turned to the role of RNA in regulating protein phase separation. FUS is an RNA binding protein, and RNA can regulate the phase behavior and material properties of FUS condensates $[40,41]$. Although recombinant FUS can undergo phase separation at low protein concentrations $(\sim 2 \mu \mathrm{M})$ in vitro, which is lower than FUS's nuclear concentration, the high level of RNA in the nucleus suppresses phase separation of FUS [42]. Our previous studies have established that RNA has a dual role in regulating the LLPS of FUS protein. At low RNA concentrations, RNA promotes FUS phase separation via the formation of "sticky" complexes, whereas at higher concentrations, it inhibits FUS condensation due to the formation of negatively charged FUSRNA complexes [41]. Since FUS-DDIT3 lacks an RNA-binding domain, we hypothesized that FUS-DDIT3 may have a lower affinity for RNA and therefore, FUS-DDIT3 condensates may be refractory to the high RNA concentrations within the nucleus. This idea is directly supported by our observations that nuclear FUS-DDIT3 forms stable condensates whereas nuclear FUS remains predominantly diffused (Fig. 1d). To test this model further, we examined the partitioning of a short RNA client ([6FAM]UGAAGGAC) into recombinant FUS and FUSDDIT3 condensates in vitro. While FUS condensates could readily interact with and enrich RNA

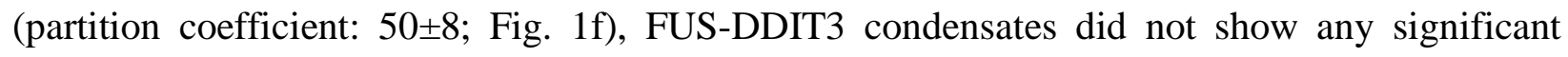
enrichment (partition coefficient: 1.60 \pm 0.03 ; Fig. 1f). We also note a subset of GFP-FUS-DDIT3 condensates exhibited hollow spherical morphologies in the nucleus (Fig. S2) similar to what was observed previously for the condensates formed by TDP43 mutants with impaired RNA binding ability [43]. Overall, these results indicate that RNA does not significantly interact with FUS-DDIT3 and provide an explanation for FUS-DDIT3's ability to form robust condensates within the RNA-rich environment of the cell nucleus where FUS primarily remains soluble.

\section{FUS-DDIT3 condensates enrich BRG1, a catalytic subunit of the mSWI/SNF complex}

Nuclear condensates can compartmentalize machineries responsible for many biochemical events such as transcription, splicing, and chromatin organization [44]. Recent studies have indicated that transcription factor condensates can activate genes by creating phase-separated transcriptional hubs at super-enhancer sites that enrich coactivators and RNA polymerase II [4547]. Based on our observations that FUS-DDIT3 forms stable nuclear condensates, we aimed to investigate if these condensates can enrich transcriptional activators/coactivators. Previous studies have established that FET fusion protein interactomes are enriched in the components of $\mathrm{mSWI} / \mathrm{SNF}$ complex and that BRG1, the key ATPase subunit that drives chromatin remodeling by $\mathrm{mSWI} / \mathrm{SNF}$, co-immunoprecipitates with the FUS-DDIT3 fusion protein [2, 22]. Therefore, we hypothesized that nuclear FUS-DDIT3 condensates may compartmentalize BRG1. To test 
this idea, we expressed GFP-tagged BRG1 along with mCherry-tagged FUS-DDIT3 in HEK293T cells. We observed that BRG1 remained diffused in the nucleus in absence of FUSDDIT3, but was readily recruited within FUS-DDIT3 nuclear condensates when co-expressed (Fig. 2a-b\&S3). Independently, using purified proteins in vitro, we observed that recombinant BRG1 protein is enriched within the FUS-DDIT3 condensates [partition coefficient: $27 \pm 7$ (Fig. 2c)]. These data suggest that phase separation of the FUS-DDIT3 leads to the enrichment and ectopic compartmentalization of the chromatin remodeler BRG1 into nuclear oncoprotein condensates.

What is the molecular mechanism of BRG1 recruitment within FUS-DDIT3 condensates? Linden et al. reported that the $\mathrm{N}$-terminal prion-like domains of FET proteins can coimmunoprecipitate with multiple subunits of the mSWI/SNF complex [2]. In addition to the PLD-containing FET oncofusions, PLD-harboring transcription activators such as EBF1 and MN1 have also been suggested to interact with BRG1 [10, 48]. We, therefore, hypothesized that the PLD of FUS-DDIT3 is responsible for recruiting BRG1 within FUS-DDIT3 condensates. To test this, we used a previously characterized OptoFUS ${ }^{\mathrm{PLD}}$ construct containing a Cry2 tag that homo-oligomerizes on exposure to blue light $(488 \mathrm{~nm})$ and nucleates formation of the FUS ${ }^{\text {PLD }}$ condensates. [49]. We observed that when co-expressed with GFP-BRG1 protein, OptoFUS ${ }^{\text {PLD }}$ droplets can enrich GFP-BRG1 (Fig. 2d \& S3). These results suggest that the PLD of FUS is sufficient to recruit and compartmentalize the chromatin remodeler BRG1 in FUS-DDIT3 condensates.

\section{Prion-like domains can act as scaffolds to recruit mSWI/SNF proteins in FUS-DDIT3 condensates}

PLDs are known to self-assemble into a variety of assemblage including phase-separated condensates, amorphous aggregates, and fibrillar solids [13, 36, 37]. Furthermore, distinct PLDs are also known to cooperate and co-condense through heterotypic PLD-PLD interactions in transcription factor condensates [11]. Given the PLD of FUS is sufficient to recruit BRG1 into FUS-DDIT3 condensates, we enquired if BRG1 also carries a prion-like domain. Sequence analysis using the PLAAC algorithm [50] revealed that the N-terminus of BRG1 (aa 1-340) is disordered and has a significant prion-like amino acid composition (Fig. S4). Therefore, it is conceivable that heterotypic PLD ${ }^{\mathrm{FUS}}$-PLD ${ }^{\mathrm{BRG} 1}$ interactions can recruit BRG1 into FUS-DDIT3 condensates. We first characterized the phase separation capability of BRG1 ${ }^{\text {PLD }}$ and observed that recombinant $\mathrm{BRG} 1^{\mathrm{PLD}}$ has a low intrinsic tendency to phase separate in vitro, only forming condensates at relatively high protein and crowder concentrations (Fig. S5a). Furthermore, when expressed in HEK293T cells, OptoBRG1 ${ }^{\text {PLD }}$ proteins did not form condensates even at concentrations that are $\sim 4$-fold higher than that required for the formation of OptoFUS ${ }^{\text {PLD }}$ condensates (Figs. 3a and S5b). Thus, the N-terminal prion-like domain of BRG1 has a weak capacity to undergo self-condensation both in vitro and in cells.

Although the PLD of BRG1 did not undergo phase separation in cells, we tested whether it could engage with the PLD of FUS through heterotypic PLD-PLD interactions. We expressed 
GFP-tagged BRG1 ${ }^{\text {PLD }}$ in cells expressing OptoFUS ${ }^{\text {PLD }}$. Upon blue light activation, cells expressing OptoFUS ${ }^{\text {PLD }}$ formed condensates of FUS ${ }^{\text {PLD }}$ that enriched GFP-BRG1 ${ }^{\text {PLD }}$ (Fig. 3b right panel). Using independent experiments, we further observed that GFP-BRG1 ${ }^{\text {PLD }}$ is enriched within the nuclear FUS-DDIT3 condensates (Fig. 3b left panel). Consistent with these results, we also found that when recombinant $\mathrm{BRG} 1^{\mathrm{PLD}}$ and $\mathrm{FUS}{ }^{\mathrm{PLD}}$ were mixed at a concentration below their individual phase separation thresholds in vitro, they form co-condensates (Fig. 3c and S6). This is likely due to heterotypic PLD-PLD interactions leading to the lowering of phase separation saturation concentration of the PLD mixture [51]. Thus, BRG1 ${ }^{\text {PLD }}$ can interact and undergo co-condensation with the prion-like domain of FUS.

A broader analysis using PLAAC revealed that in addition to BRG1, multiple subunits of the mSWI/SNF complex contain bona fide PLDs (Fig. 3d and S4). These include SMARCC1 and SMARCC2, which along with BRG1, are three of the four core subunits of the complex that orchestrate chromatin remodeling [52, 53]. In addition, accessory (and/or signature) subunits such as ARID1A and ARID1B, mutations of which are highly correlated with oncogenic transformation [23, 54] also have long tracts of prion-like sequences (Fig. 3d and S4). To test if these PLDs could also mediate interactions with FET fusion oncoproteins, we reconstituted FUSDDIT3 condensates in vitro and looked for enrichment of fluorescently labeled PLDs from the three associated factors with the longest PLDs: ARID1A, ARID1B, and SS18. All of the tested PLDs were enriched within FUS-DDIT3 condensates (Fig. 3e), although with varied partition coefficients (partition coefficient ranges from $\sim 4$ to 40 ), suggesting different strengths of their interactions. This can be attributed to distinct sequence compositions, chain lengths, and charge patterns of the tested PLDs. Together, these data suggest that the prion-like domain of FET fusion oncoproteins can engage with prion-like domains of multiple mSWI/SNF proteins and recruit them to ectopic nuclear condensates.

\section{FUS-DDIT3 forms condensates on dsDNA and recruits chromatin remodeler BRG1}

Our results discussed above suggest that the PLD of FUS enables phase separation of the DDIT3 transcription factor. Transcription factors can interact with the surface of DNA and such interactions can contribute to their phase separation at specific genomic loci $[19,55,56]$. To test if FUS-DDIT3 can form condensates on DNA, we tethered a single double-stranded (ds) $\lambda$-phage genomic DNA between two optically trapped polystyrene beads using laminar-flow in a microfluidic glass chamber (see Materials \& Methods for further details and Figs. 4a\&S7). Upon transferring this tethered DNA molecule into a channel containing $250 \mathrm{nM}$ AlexaFluor488labeled FUS-DDIT3, we detected the formation of distinct FUS-DDIT3 clusters on the dsDNA (Fig 4b, top left panel). The FUS-DDIT3 clusters formed at multiple loci on a single DNA chain. This is likely because FUS-DDIT3 interacts promiscuously with the $\lambda$-phage genomic DNA [19]. Next, to determine if these FUS-DDIT3 condensates can recruit BRG1, the single-molecule DNA tether with FUS-DDIT3 condensates was transferred to a microfluidic channel containing $10 \mathrm{nM}$ of RED-tris-NTA-labeled BRG1 (Fig. S7). In line with our above-mentioned results, we observed that the BRG1 co-localizes with the FUS-DDIT3 condensates on the surface of dsDNA (Fig. 4b, top right panel). The co-localization of BRG1 within FUS-DDIT3 condensates was 
confirmed using the confocal images as well as by analyzing the intensity profiles which revealed a clear overlap of FUS-DDIT3 and BRG1 peaks (Fig. 4b bottom panel). We also noted a few isolated BRG1 peaks that are present across the dsDNA without any detectable spatial overlap with FUS-DDIT3 condensates and the occurrence of such peaks increases with increasing bulk concentration of BRG1 from $10 \mathrm{nM}$ to $50 \mathrm{nM}$ (Fig. S8). Such foci formation may indicate the presence of a possible interaction between BRG1 and the $\lambda$-phage genomic DNA that is independent of FUS-DDIT3.

\section{Many recurrent oncogenic translocations result in the fusion of prion-like domains with transcriptional regulators}

Our results, presented so far, indicate that both partners in the fusion pair (i.e., FUS ${ }^{\mathrm{PLD}}$ and DDIT3) are likely to contribute to the neomorphic activity of the FET oncofusion protein - the DDIT3 $^{\text {DBD }}$ can recruit the fusion transcription factor to specific genomic loci, whereas the FUS $^{\text {PLD }}$ can drive their condensation and the subsequent recruitment of the chromatin remodeler mSWI/SNF. Since many transcription factors are involved in oncogenic fusions [57], we asked whether fusion between the DNA localization domain of transcriptional regulators and PLD of prion-containing proteins represents a generic category of oncogenic transcription regulators. To answer this, we focused on translocations involving transcription regulators. Specifically, we retrieved sequences of transcription regulator fusions that are recurrently present in cancer patients using the following selection criteria: $(i)$ present in at least 25 curated patient samples in the COSMIC database [58], and (ii) present in $>10 \%$ of tumor samples in a given malignancy (The list of recurrent translocations involving transcriptional regulators was obtained from the reference [59]). We further narrowed down our list to the oncofusions that harbor at least one prion-like domain and identified a total of 16 different recurrent oncofusions involving 10 fusion pairs (Table 1). These proteins represent in-frame fusions between a prion-like domain and a specific DNA localization domain. The DNA localization domains were either a specific DNAbinding domain (e.g., ERG ${ }^{\mathrm{DBD}}$ in FUS-ERG fusion) or a protein-protein interaction motif that specifically interacts with transcription regulators (e.g., SSX ${ }^{\mathrm{SSXRD}}$ in SS18-SSX family fusions). In all these oncofusions, prion-like domains and DNA-localization domains originate from the two separate genes involved in respective in-frame genomic translocations. Not surprisingly, we find that many of these recurrent oncofusions have already been implicated in chromatin reorganization (Table 1). Therefore, as observed for FUS-DDIT3 fusion protein in our experiments, other PLD-containing oncogenic transcription regulators may similarly engage with $\mathrm{mSWI} / \mathrm{SNF}$ chromatin remodelers to drive aberrant transcriptional outcomes via protein cocondensation. 


\section{Discussion}

A major consequence of FET oncofusions is the transfer of the prion-like domain from an RNAbinding protein to a DNA-binding protein. Our results described here along with two recent reports suggest that FET-fusion oncoproteins can form ectopic nuclear condensates [18, 19]. These reports also suggest that FET-fusion protein condensates can activate transcription at DNA enhancer sites by engaging with essential transcriptional co-activators such as BRD4 and by recruiting RNA polymerase II [18, 19]. In our study, we focus on characterizing the interactions between FET-fusion condensates with the ATP-dependent chromatin remodeler complex $\mathrm{mSWI} / \mathrm{SNF}$. Chromatin remodeling is one of the primary and essential steps in the regulation of gene expression and the aberrant targeting of $\mathrm{mSWI} / \mathrm{SNF}$ complexes can be detrimental to physiologic cellular processes [60]. Our results reveal that FUS-DDIT3 condensates can compartmentalize BRG1, a key catalytic subunit (ATPase) of $\mathrm{mSWI} / \mathrm{SNF}$ that is responsible for ATP-dependent remodeling of DNA-histone interactions. Since a continuous activity of the $\mathrm{mSWI} / \mathrm{SNF}$ is often required to maintain the appropriate chromatin state at the target genomic locus [61, 62], the selective enrichment of mSWI/SNF components by FUS-DDIT3 condensates provides two possible routes to transcriptional reprograming (Fig. 5). First, condensates of FETfusion proteins formed at ectopic genomic regions, as specified by binding sites of the DNAbinding domain, can recruit BRG1 and modify local chromatin folding to activate transcription (Fig. 5, left panel). Previous studies with FET oncoproteins, such as EWS-FLI1 and FUS-DDIT3 showed retargeting of chromatin remodelers to microsatellites and enhancers respectively [20, 63], lending support to this model. Congruently, condensates formed by both FET proteins and FET fusion-oncoproteins are capable of enhancing gene transcription [19, 64]. Alternatively, we propose a model where sequestration of $\mathrm{mSWI} / \mathrm{SNF}$ subunits away from their natural targets and trapping them in ectopic condensates can contribute to a loss-of-function phenotype (Fig. 5, right panel). This model is consistent with the recently reported observations that $\mathrm{mSWI} / \mathrm{SNF}$ complex suppresses the H3K27Me3 histone modification under normal conditions, but when FET oncoproteins are expressed, the H3K27Me3 levels within the cells are upregulated likely due to the sequestration of $\mathrm{mSWI} / \mathrm{SNF}$ away from its physiological target sites [2].

At the molecular level, the presence of PLDs in multiple subunits of the mSWI/SNF (Fig. S4) suggests that PLD-mediated interactions could be a generic mechanism for the recruitment of these complexes to their target sites. This is based on our observations that the PLD of FUS is sufficient to target the mSWI/SNF ATPase, BRG1, to FUS-DDIT3 condensates (Figs. 2\&3). Moreover, the PLDs of $\mathrm{mSWI} / \mathrm{SNF}$ proteins can synergistically engage with FET $^{\mathrm{PLD}}$ and reduce the phase separation threshold as observed in mixtures of FUS ${ }^{\text {PLD }}$ and BRG1 ${ }^{\text {PLD }}$ (Fig. S6). These data also indicate that heterotypic PLD-mediated co-condensation may play a fundamental role in the functional assembly of the mSWI/SNF complex itself.

We envision that hijacking of mSWI/SNF complex by FET oncofusions through heterotypic PLD-PLD interactions is a generic strategy employed by many aberrant transcription regulators. This is supported by our analysis that many recurrent oncogenic translocations involve the fusion of a prion-like domain to a DNA recruitment domain and result in a widespread reorganization of the chromatin landscape (Table 1). In addition to the FET 
oncofusions, SS18-SSX fusions represent an interesting category of oncogenic translocations. In SS18-SSX fusions, the prion-like domain of SS18 is fused to the C-terminal segments of SSX family proteins containing the SSXRD domain $[65,66]$. SSXRD domain is a protein-protein interaction module that directly interacts with the polycomb complex DNA-binding protein, KDM2B [67]. Not surprisingly, SS18-SSX fusions recruit mSWI/SNF complex to KDM2Bbinding genomic loci and result in the aberrant activation of numerous otherwise repressed genes $[67,68]$. Therefore, similar to the FUS ${ }^{\mathrm{PLD}}$, it is likely that $\mathrm{SS} 18^{\mathrm{PLD}}$ is capable of recruiting $\mathrm{mSWI} / \mathrm{SNF}$ chromatin remodelers via SS18${ }^{\mathrm{PLD}}-\mathrm{SWI} / \mathrm{SNF}^{\mathrm{PLD}}$ interactions. However, unlike FET fusions where the neomorphic transcriptional activator is recruited to specific genomic locations via DNA-binding domains fused to the $\mathrm{FET}^{\mathrm{PLD}}$, SS18-SSX fusions may utilize specific proteinprotein interaction module from SSX family members (i.e, the SSXRD domain) to recruit oncofusion proteins to specific loci.

In summary, our results provide a molecular mechanism with regards to how FET oncofusions can synergistically engage with the ATP-dependent chromatin remodeler $\mathrm{mSWI}$ SNF. Our bioinformatics analysis reveals that PLD fusions to DNA-binding domains go beyond FET fusion oncoproteins and the proposed role of heterotypic PLD-PLD interactions in recruiting $\mathrm{mSWI} / \mathrm{SNF}$ complex at non-native genomic loci may play a central role in all such cases. Future studies can test the generality of this model and subsequently target the phase separation and/or mSWI/SNF complex engagement capacities of PLD containing oncofusion proteins for potential cancer therapeutics. 


\section{References}

1. Panagopoulos, I., et al., Fusion of the EWS and CHOP genes in myxoid liposarcoma. Oncogene, 1996. 12(3): p. 489-494.

2. Lindén, M., et al., FET family fusion oncoproteins target the SWI/SNF chromatin remodeling complex. EMBO reports, 2019. 20(5): p. e45766.

3. Bunting, S.F. and A. Nussenzweig, End-joining, translocations and cancer. Nature reviews Cancer, 2013. 13(7): p. 443-454.

4. Perry, J.A., B.K.A. Seong, and K. Stegmaier, Biology and therapy of dominant fusion oncoproteins involving transcription factor and chromatin regulators in sarcomas. Annual Review of Cancer Biology, 2019. 3: p. 299-321.

5. Mitelman, F., B. Johansson, and F. Mertens, The impact of translocations and gene fusions on cancer causation. Nature Reviews Cancer, 2007. 7(4): p. 233-245.

6. Alberti, S., et al., A Systematic Survey Identifies Prions and Illuminates Sequence Features of Prionogenic Proteins. Cell, 2009. 137(1): p. 146-158.

7. March, Z.M., O.D. King, and J. Shorter, Prion-like domains as epigenetic regulators, scaffolds for subcellular organization, and drivers of neurodegenerative disease. Brain research, 2016. 1647: p. 9-18.

8. Gotor, N.L., et al., RNA-binding and prion domains: the Yin and Yang of phase separation. Nucleic acids research, 2020. 48(17): p. 9491-9504.

9. Franzmann, T.M., et al., Phase separation of a yeast prion protein promotes cellular fitness. Science, 2018. 359(6371).

10. Wang, Y., et al., A Prion-like Domain in Transcription Factor EBF1 Promotes Phase Separation and Enables B Cell Programming of Progenitor Chromatin. Immunity, 2020. 53(6): p. 1151-1167. e6.

11. Frazer, C., et al., Epigenetic cell fate in Candida albicans is controlled by transcription factor condensates acting at super-enhancer-like elements. Nature Microbiology, 2020. 5(11): p. 1374-1389.

12. Martin, E.W., et al., Valence and patterning of aromatic residues determine the phase behavior of prion-like domains. Science, 2020. 367(6478): p. 694-699.

13. Wang, J., et al., A molecular grammar governing the driving forces for phase separation of prion-like RNA binding proteins. Cell, 2018. 174(3): p. 688-699. e16.

14. Keegan, L., G. Gill, and M. Ptashne, Separation of DNA binding from the transcriptionactivating function of a eukaryotic regulatory protein. Science, 1986. 231(4739): p. 699704.

15. Triezenberg, S.J., Structure and function of transcriptional activation domains. Current opinion in genetics \& development, 1995. 5(2): p. 190-196.

16. Liou, S.-H., et al., Structure of the p53/RNA polymerase II assembly. Communications Biology, 2021. 4(1): p. 1-12.

17. Keaveney, M. and K. Struhl, Activator-mediated recruitment of the RNA polymerase II machinery is the predominant mechanism for transcriptional activation in yeast. Molecular cell, 1998. 1(6): p. 917-924.

18. Owen, I., et al., The oncogenic transcription factor FUS-CHOP can undergo nuclear liquid-liquid phase separation. bioRxiv, 2021.

19. Zuo, L., et al., Loci-specific phase separation of FET fusion oncoproteins promotes gene transcription. Nature Communications, 2021. 12(1): p. 1491. 
20. Boulay, G., et al., Cancer-specific retargeting of BAF complexes by a prion-like domain. Cell, 2017. 171(1): p. 163-178. e19.

21. Riggi, N., et al., EWS-FLI1 utilizes divergent chromatin remodeling mechanisms to directly activate or repress enhancer elements in Ewing sarcoma. Cancer Cell, 2014. 26(5): p. 668-681.

22. Jamie, S., et al., The FUS-DDIT3 interactome in myxoid liposarcoma. Neoplasia, 2019. 21(8): p. 740-751.

23. Kadoch, C. and G.R. Crabtree, Mammalian SWI/SNF chromatin remodeling complexes and cancer: Mechanistic insights gained from human genomics. Science advances, 2015. 1(5): p. e1500447.

24. Mashtalir, N., et al., Modular organization and assembly of SWI/SNF family chromatin remodeling complexes. Cell, 2018. 175(5): p. 1272-1288. e20.

25. Tolstorukov, M.Y., et al., Swi/Snf chromatin remodeling/tumor suppressor complex establishes nucleosome occupancy at target promoters. Proceedings of the National Academy of Sciences, 2013. 110(25): p. 10165-10170.

26. Alver, B.H., et al., The SWI/SNF chromatin remodelling complex is required for maintenance of lineage specific enhancers. Nature Communications, 2017. 8(1): p. 14648.

27. Xu, F., S. Flowers, and E. Moran, Essential role of ARID2 protein-containing SWI/SNF complex in tissue-specific gene expression. J Biol Chem, 2012. 287(7): p. 5033-41.

28. Yudkovsky, N., et al., Recruitment of the SWI/SNF chromatin remodeling complex by transcriptional activators. Genes \& development, 1999. 13(18): p. 2369-2374.

29. Kadoch, C., et al., Proteomic and bioinformatic analysis of mammalian SWI/SNF complexes identifies extensive roles in human malignancy. Nature genetics, 2013. 45(6): p. 592-601.

30. Shain, A.H. and J.R. Pollack, The spectrum of SWI/SNF mutations, ubiquitous in human cancers. PloS one, 2013. 8(1): p. e55119.

31. Neely, K.E., et al., Activation domain-mediated targeting of the SWI/SNF complex to promoters stimulates transcription from nucleosome arrays. Molecular cell, 1999. 4(4): p. 649-655.

32. Vierbuchen, T., et al., AP-1 Transcription Factors and the BAF Complex Mediate SignalDependent Enhancer Selection. Molecular Cell, 2017. 68(6): p. 1067-1082.e12.

33. Panagopoulos, I., et al., Characterization of the $\langle$ em $>C H O P</ e m>$ Breakpoints and Fusion Transcripts in Myxoid Liposarcomas with the 12;16 Translocation. Cancer Research, 1994. 54(24): p. 6500-6503.

34. Crozat, A., et al., Fusion of CHOP to a novel RNA-binding protein in human myxoid liposarcoma. Nature, 1993. 363(6430): p. 640-644.

35. Rabbitts, T., et al., Fusion of the dominant negative transcription regulator CHOP with a novel gene FUS by translocation $t(12 ; 16)$ in malignant liposarcoma. Nature genetics, 1993. 4(2): p. 175-180.

36. Patel, A., et al., A liquid-to-solid phase transition of the ALS protein FUS accelerated by disease mutation. Cell, 2015. 162(5): p. 1066-1077.

37. Kato, M., et al., Cell-free formation of RNA granules: low complexity sequence domains form dynamic fibers within hydrogels. Cell, 2012. 149(4): p. 753-767.

38. Schmit, J.D., et al., Protein network structure enables switching between liquid and gel states. Journal of the American Chemical Society, 2019. 142(2): p. 874-883. 
39. Bouchard, J.J., et al., Cancer mutations of the tumor suppressor SPOP disrupt the formation of active, phase-separated compartments. Molecular cell, 2018. 72(1): p. 1936. e8.

40. Alshareedah, I., et al., Interplay between short-range attraction and long-range repulsion controls reentrant liquid condensation of ribonucleoprotein-RNA complexes. Journal of the American Chemical Society, 2019. 141(37): p. 14593-14602.

41. Banerjee, P.R., et al., Reentrant phase transition drives dynamic substructure formation in ribonucleoprotein droplets. Angewandte Chemie, 2017. 129(38): p. 11512-11517.

42. Maharana, S., et al., RNA buffers the phase separation behavior of prion-like RNA binding proteins. Science, 2018. 360(6391): p. 918-921.

43. $\mathrm{Yu}, \mathrm{H}$., et al., HSP70 chaperones RNA-free TDP-43 into anisotropic intranuclear liquid spherical shells. Science, 2021. 371(6529).

44. Sabari, B.R., A. Dall'Agnese, and R.A. Young, Biomolecular Condensates in the Nucleus. Trends in Biochemical Sciences, 2020. 45(11): p. 961-977.

45. Boija, A., et al., Transcription factors activate genes through the phase-separation capacity of their activation domains. Cell, 2018. 175(7): p. 1842-1855. e16.

46. Sabari, B.R., et al., Coactivator condensation at super-enhancers links phase separation and gene control. Science, 2018. 361(6400): p. eaar3958.

47. Cho, W.-K., et al., Mediator and RNA polymerase II clusters associate in transcriptiondependent condensates. Science, 2018. 361(6400): p. 412-415.

48. $\quad$ Riedel, S.S., et al., SWI/SNF Dysregulation through a Prion-like Domain Causes AML. 2019, American Society of Hematology Washington, DC.

49. Shin, Y., et al., Spatiotemporal control of intracellular phase transitions using lightactivated optoDroplets. Cell, 2017. 168(1-2): p. 159-171. e14.

50. Lancaster, A.K., et al., PLAAC: $a$ web and command-line application to identify proteins with prion-like amino acid composition. Bioinformatics, 2014. 30(17): p. 2501-2502.

51. Ruff, K.M., F. Dar, and R.V. Pappu, Ligand effects on phase separation of multivalent macromolecules. Proceedings of the National Academy of Sciences, 2021. 118(10).

52. Mani, U., et al., SWI/SNF Infobase-An exclusive information portal for SWI/SNF remodeling complex subunits. PLoS One, 2017. 12(9): p. e0184445.

53. Phelan, M.L., et al., Reconstitution of a core chromatin remodeling complex from SWI/SNF subunits. Molecular cell, 1999. 3(2): p. 247-253.

54. Davoli, T., et al., Cumulative haploinsufficiency and triplosensitivity drive aneuploidy patterns and shape the cancer genome. Cell, 2013. 155(4): p. 948-962.

55. Morin, J.A., et al., Surface condensation of a pioneer transcription factor on DNA. bioRxiv, 2020.

56. Renger, R., et al., Co-condensation of proteins with single-and double-stranded DNA. bioRxiv, 2021.

57. Kim, P., L.Y. Ballester, and Z. Zhao, Domain retention in transcription factor fusion genes and its biological and clinical implications: a pan-cancer study. Oncotarget, 2017. 8(66): p. 110103-110117.

58. Tate, J.G., et al., COSMIC: the Catalogue Of Somatic Mutations In Cancer. Nucleic Acids Research, 2019. 47(D1): p. D941-D947.

59. Brien, G.L., K. Stegmaier, and S.A. Armstrong, Targeting chromatin complexes in fusion protein-driven malignancies. Nat Rev Cancer, 2019. 19(5): p. 255-269. 
60. McBride, M.J. and C. Kadoch, Disruption of mammalian SWI/SNF and polycomb complexes in human sarcomas: mechanisms and therapeutic opportunities. The Journal of pathology, 2018. 244(5): p. 638-649.

61. Schick, S., et al., Acute BAF perturbation causes immediate changes in chromatin accessibility. Nature Genetics, 2021: p. 1-10.

62. Iurlaro, M., et al., Mammalian SWI/SNF continuously restores local accessibility to chromatin. Nature Genetics, 2021: p. 1-9.

63. Chen, M., et al., Disruption of oncogenic targeting by ISWI via phosphorylation of a prion-like domain. bioRxiv, 2020: p. 2020.03.11.987750.

64. Wei, M.-T., et al., Nucleated transcriptional condensates amplify gene expression. Nature Cell Biology, 2020. 22(10): p. 1187-1196.

65. Lim, F.L., et al., A KRAB-related domain and a novel transcription repression domain in proteins encoded by SSX genes that are disrupted in human sarcomas. Oncogene, 1998. 17(15): p. 2013-8.

66. Skytting, B., et al., A Novel Fusion Gene, SYT-SSX4, in Synovial Sarcoma. JNCI: Journal of the National Cancer Institute, 1999. 91(11): p. 974-975.

67. Banito, A., et al., The SS18-SSX Oncoprotein Hijacks KDM2B-PRC1.1 to Drive Synovial Sarcoma. Cancer Cell, 2018. 33(3): p. 527-541 e8.

68. Boulay, G., et al., The chromatin landscape of primary synovial sarcoma organoids is linked to specific epigenetic mechanisms and dependencies. Life Sci Alliance, 2021. 4(2).

69. Boulay, G., et al., Cancer-Specific Retargeting of BAF Complexes by a Prion-like Domain. Cell, 2017. 171(1): p. 163-178 e19.

70. Linden, M., et al., FET family fusion oncoproteins target the SWI/SNF chromatin remodeling complex. EMBO Rep, 2019. 20(5).

71. Gryder, B.E., et al., PAX3-FOXO1 Establishes Myogenic Super Enhancers and Confers BET Bromodomain Vulnerability. Cancer Discovery, 2017. 7(8): p. 884.

72. Sunkel, B., et al., Pioneer activity of an oncogenic fusion transcription factor at inaccessible chromatin. bioRxiv, 2020: p. 2020.12.11.420232.

73. Nagai, M., et al., Analysis of transforming activity of human synovial sarcoma-associated chimeric protein SYT-SSX1 bound to chromatin remodeling factor hBRM/hSNF2 alpha. Proc Natl Acad Sci U S A, 2001. 98(7): p. 3843-8. 


\section{Data Availability}

All data relevant to the findings of this manuscript are included in the manuscript and the supplementary appendix. Additional data are available from the authors upon reasonable request.

\section{Acknowledgment}

The authors acknowledge Dr. Sarah Walker of the University at Buffalo, SUNY for valuable help with protein purification. P.R.B. acknowledges the College of Arts and Sciences at the University at Buffalo, SUNY, and the National Institute of General Medical Sciences (NIGMS) of the National Institutes of Health (R35 GM138186) for financial support.

\section{Author Contributions}

P.R.B. and M.M.M. conceived the idea. P.R.B., M.M.M., R.D., and T.K. designed the study. R.D. and T.K. performed the experiments and data analysis. M.M.M. performed the bioinformatics analysis. All authors contributed to writing the manuscript.

\section{Competing Interests}

The authors declare no competing interests. 
a

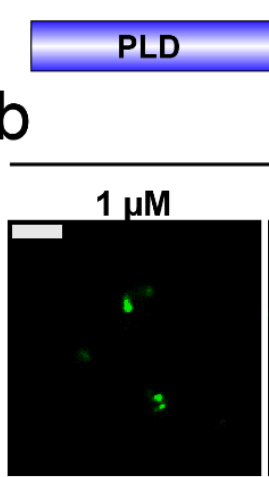

$$
\text { C } t=0 s
$$

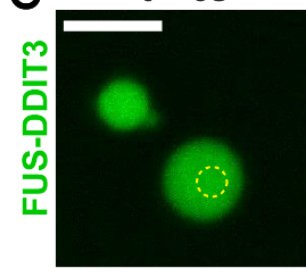

d

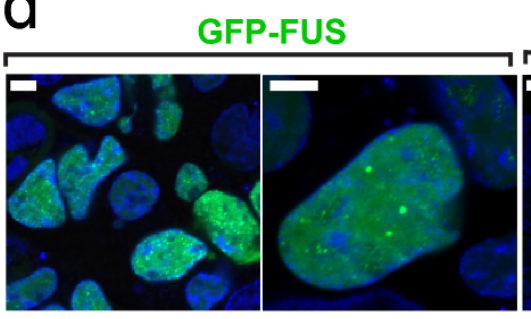

e

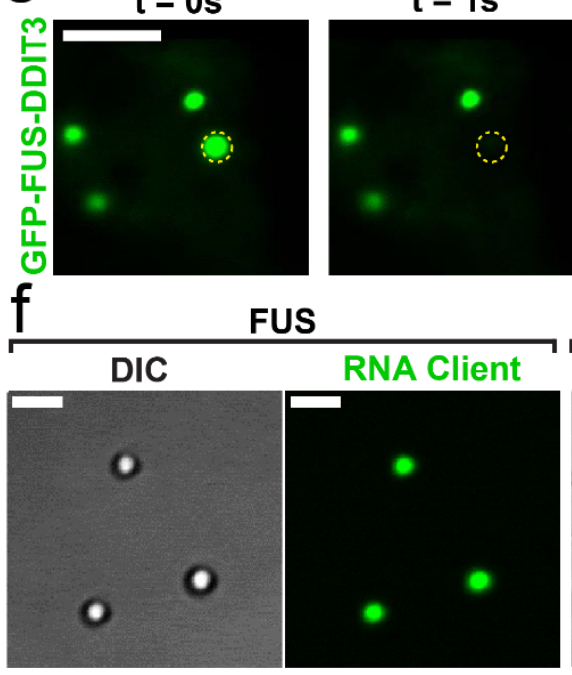

FUS

RBD
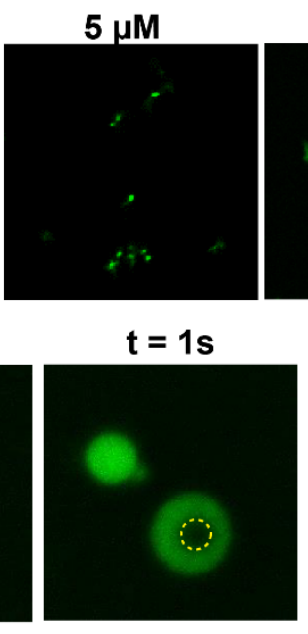

DDIT3

DBD
FUS-DDIT3 Fusion

PLD

FUS-DDIT3
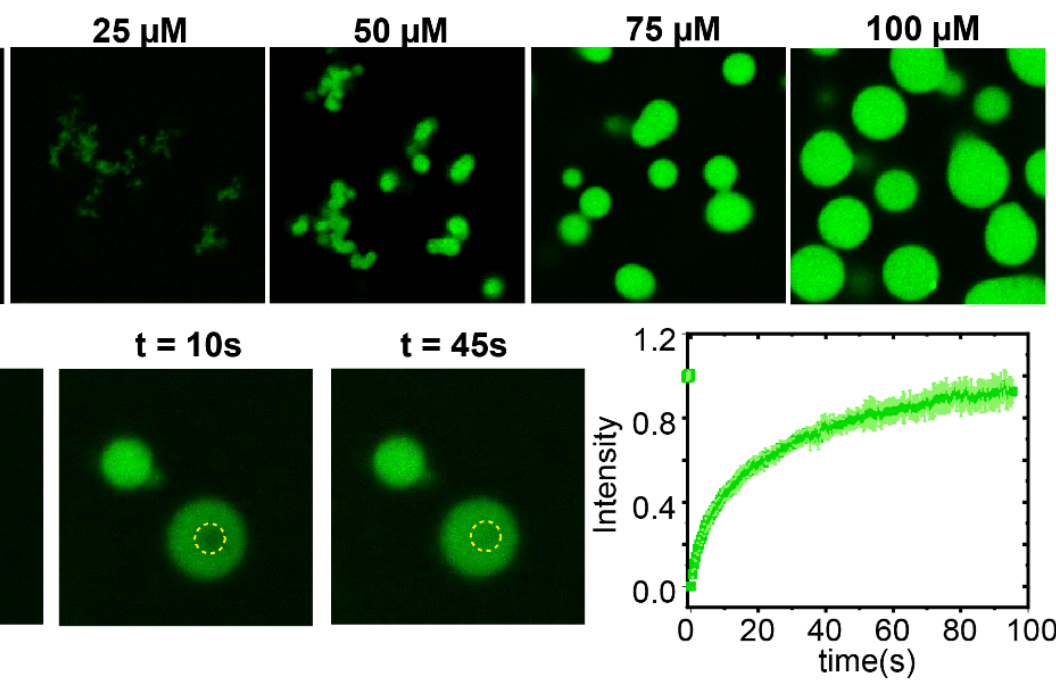

GFP-FUS-DDIT3

GFP-DDIT3
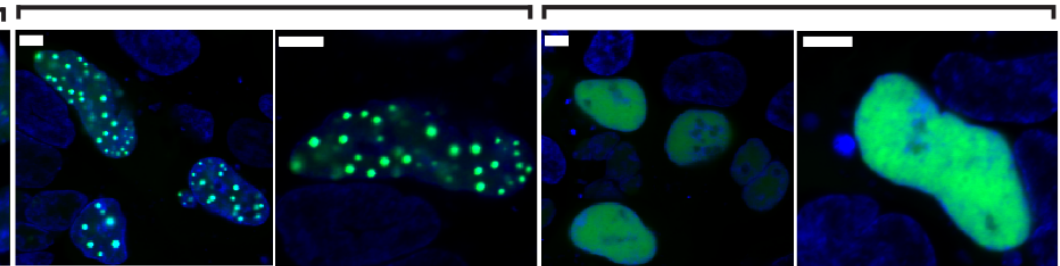

$t=10 s$
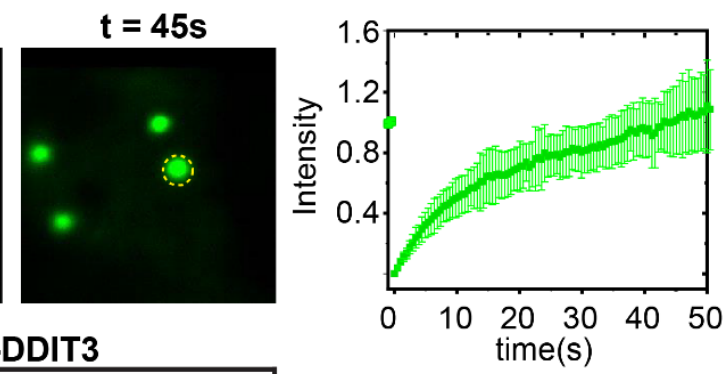

FUS-DDIT3

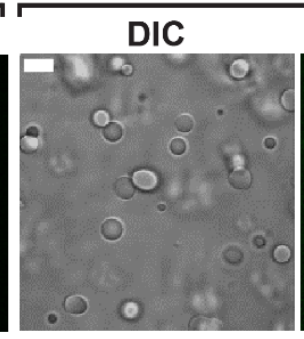

RNA Client

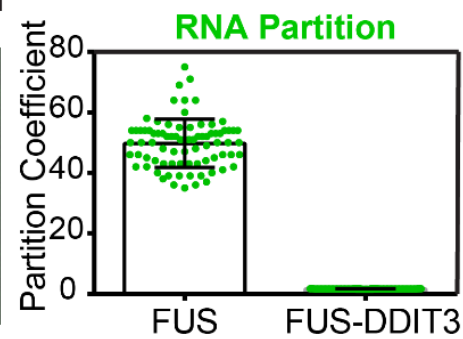


Figure 1. Oncofusion protein FUS-DDIT3 undergoes liquid-liquid phase separation in vitro and in mammalian cells. (a) Schematics of FUS, DDIT3, and FUS-DDIT3 domain architectures (PLD: Prion-like domain; RBD: RNA-binding domain; DBD: DNA-binding domain). (b) Fluorescence microscopy images of the assemblies formed by recombinantly purified FUS-DDIT3 (mixed with $250 \mathrm{nM}$ AlexaFluor488-labeled FUS-DDIT3) at varying protein concentrations. (c) Representative fluorescence recovery after photobleaching (FRAP) images of recombinant FUS-DDIT3 condensates at $50 \mu \mathrm{M}(\mathrm{t}=0 \mathrm{~s}$ : pre-bleach; $\mathrm{t}=1 \mathrm{~s}$ : bleach; $\mathrm{t}>1$ s: recovery). Right: The FRAP curve shows the average intensity and standard deviation of the intensity profiles over time $(n=3)$. (d) Fluorescence microscopy images of HEK293T cells expressing GFP-tagged proteins (FUS, FUS-DDIT3, or DDIT3), as indicated. Hoechst was used to stain the nucleus and is shown in blue. (e) Representative fluorescence recovery after photobleaching (FRAP) images of GFP-FUS-DDIT3 condensates expressed in HEK293T cells $(\mathrm{t}=0$ s: pre-bleach; $\mathrm{t}=1 \mathrm{~s}$ : bleach; $\mathrm{t}>1 \mathrm{~s}$ : recovery). Right: The FRAP curve shows the average intensity and standard deviation of the intensity profiles over time $(n=3)$. (f) Partitioning of FAM-labeled RNA client into condensates of recombinant FUS-DDIT3 $(50 \mu \mathrm{M})$ and recombinant FUS $(6 \mu \mathrm{M})$. (DIC: Differential Interference Contrast; FAM: 6Carboxyfluorescein). Right: Enrichment is calculated as partition coefficients. Mean and standard deviation are shown. The scale bar is $5 \mu \mathrm{m}$ for all images. 

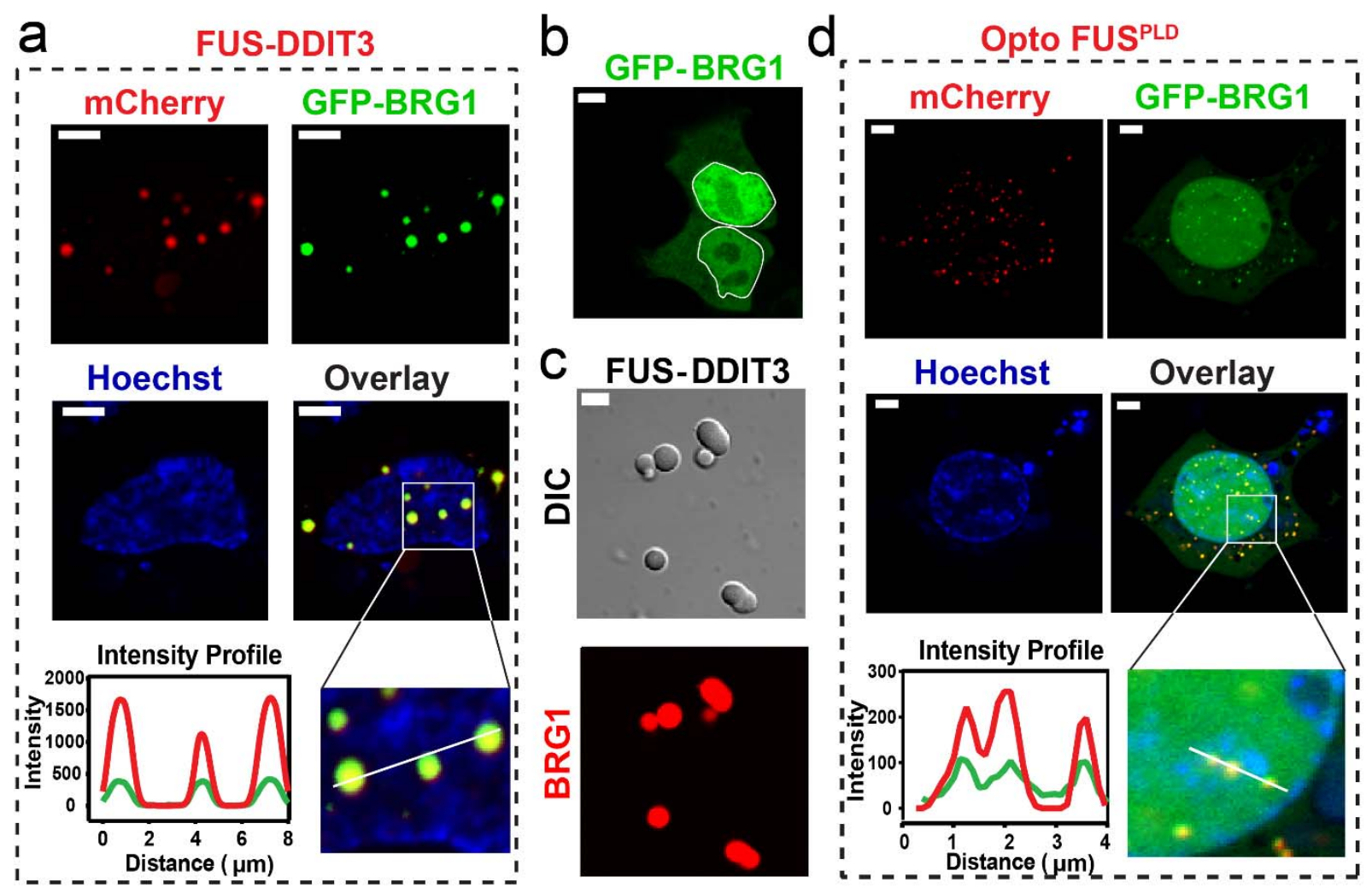

Figure 2. FUS-DDIT3 condensates enrich mSWI/SNF subunit BRG1 via the FUS prion-like $^{\text {iat }}$ domain . (a) HEK293T cells co-expressing GFP-BRG1 and Cry2-mCherry-FUS-DDIT3. FUSDDIT3 condensates were formed via protein overexpression without any blue-light activation and co-localized with GFP-BRG1 (see Materials \& Methods). Hoechst (blue) was used to stain the nucleus. The region demarcated in the white square is magnified and the fluorescence intensity profiles are shown across the linear section (white line). Green represents the intensity profile of GFP-BRG1 and red represents the profile for Cry2-mCherry-FUS-DDIT3. (b) HEK293T cells expressing the GFP-BRG1 protein show diffused distribution in both nucleus (demarcated by the white line) and the cytoplasm. (c) Partitioning of RED-tris-NTA-labeled BRG1 $(0.5 \mu \mathrm{M})$ into recombinant FUS-DDIT3 condensates $(50 \mu \mathrm{M})$. (d) HEK293T cells coexpressing GFP-BRG1 and Cry2-mCherry-FUS ${ }^{\text {PLD }}\left(\right.$ OptoFUS $^{\text {PLD }}$ ). OptoFUS ${ }^{\text {PLD }}$ droplets were formed by activating with blue light for sixty seconds and then enrichment of GFP-BRG1 was analyzed within these light-induced condensates. Hoechst (blue) was used to stain the nucleus. The region demarcated in the white square is magnified and the fluorescence intensity profiles are shown across the linear section (white line). Green represents the intensity profile of GFPBRG1 and red represents the profile for Cry2-mCherry-FUS ${ }^{\mathrm{PLD}}$. The scale bar is $5 \mu \mathrm{m}$ for all images. 
a
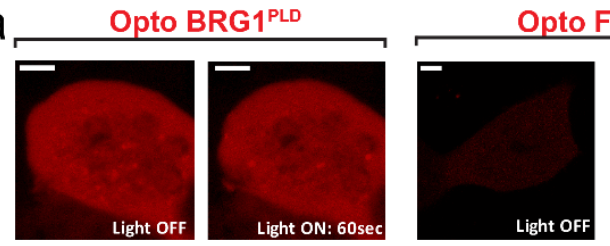

FUS-DDIT3

Opto FUS ${ }^{P L D}$

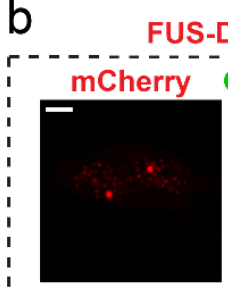

Hoechst

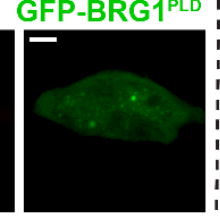

Overlay
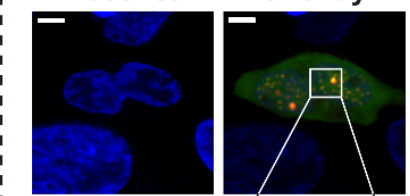

Intensity Profile : 量 50 .

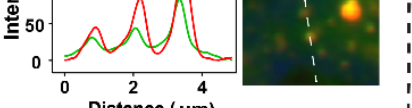

Distance $(\mu \mathrm{m})$

BRG1PLD

$(75 \mu \mathrm{M})$

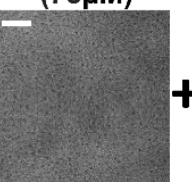

FUS ${ }^{P L D}$

$(50 \mu \mathrm{M})$
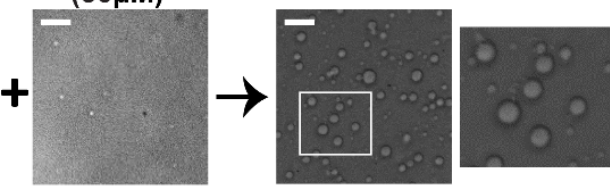
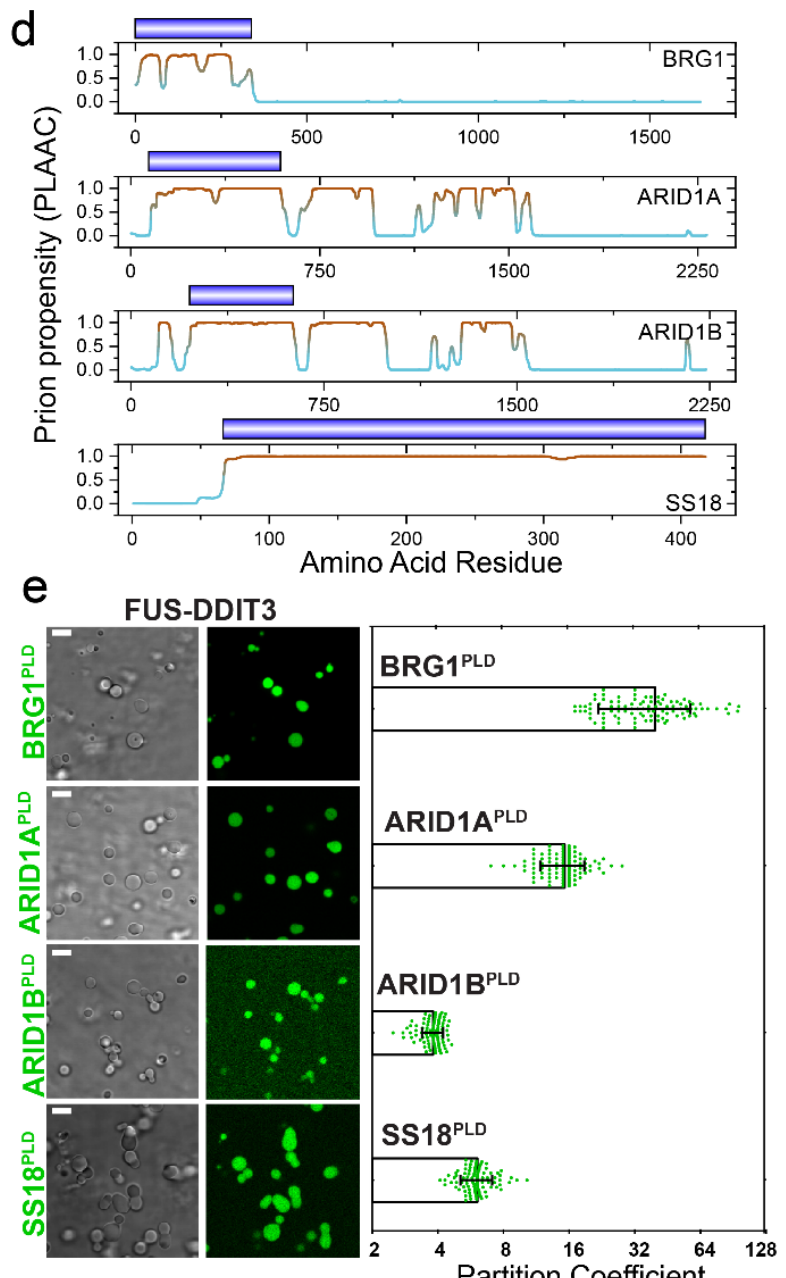

Figure 3. Many mSWI/SNF complex subunits contain PLDs and enrich into FUS-DDIT3 condensates via heterotypic PLD-PLD interactions. (a) HEK293T cell expressing OptoBRG1 $^{\text {PLD }}$ (left) or OptoFUS ${ }^{\mathrm{PLD}}$ (right) before and after activation with blue light for 60 seconds. Scale bar is $5 \mu \mathrm{m}$ (b) HEK293T cells co-expressing GFP-BRG1 ${ }^{\text {PLD }}$ and either Cry2mCherry-FUS-DDIT3 (left) or OptoFUS ${ }^{\text {PLD }}$ (right). OptoFUS ${ }^{\text {PLD }}$ droplets were formed by blue light stimulation for sixty seconds and then enrichment of GFP-BRG1 ${ }^{\text {PLD }}$ was analyzed within the condensates. Cry2-mCherry-FUS-DDIT3 condensates were formed via protein overexpression without any blue-light activation. Hoechst (blue) was used to stain the nucleus. The region demarcated in the white square is magnified and the fluorescence intensity profile is shown across the linear section (white line). Green represents the intensity profile of GFPBRG1 ${ }^{\text {PLD }}$ and red represents the profile for either Cry2-mCherry-FUS-DDIT3 or OptoFUS ${ }^{\text {PLD }}$. The scale bar is $5 \mu \mathrm{m}$. (c) Co-condensation of purified BRG1 ${ }^{\mathrm{PLD}}$ and FUS ${ }^{\mathrm{PLD}}$. No droplets were observed before mixing. The region demarcated in the white square is magnified and shown for better clarity. The scale bar is $10 \mu \mathrm{m}$. (d) PLAAC analysis showing multiple regions with high prion-propensity for the four selected subunits of mSWI/SNF complex (see Fig. S4 for PLAAC profiles for all PLD-containing mSWI/SNF complex subunits). Domains corresponding to royal blue bars were recombinantly expressed and purified and used in our experiments (panel e). (e) 
Partitioning of recombinant PLDs from panel $d$ (fluorescently labeled with AlexaFluor488) into FUS-DDIT3 condensates $(50 \mu \mathrm{M})$. Enrichment is calculated as partition coefficient. Mean and standard deviation are shown. (Partition coefficients: $B R G 1^{\text {PLD }}=40 \pm 20$; ARID1A $^{\text {PLD }}=15 \pm 4$; $\left.\mathrm{ARID} 1 \mathrm{~B}^{\mathrm{PLD}}=3.8 \pm 0.4 ; \mathrm{SS} 18^{\mathrm{PLD}}=6 \pm 1\right)$. The scale bar is $10 \mu \mathrm{m}$. 

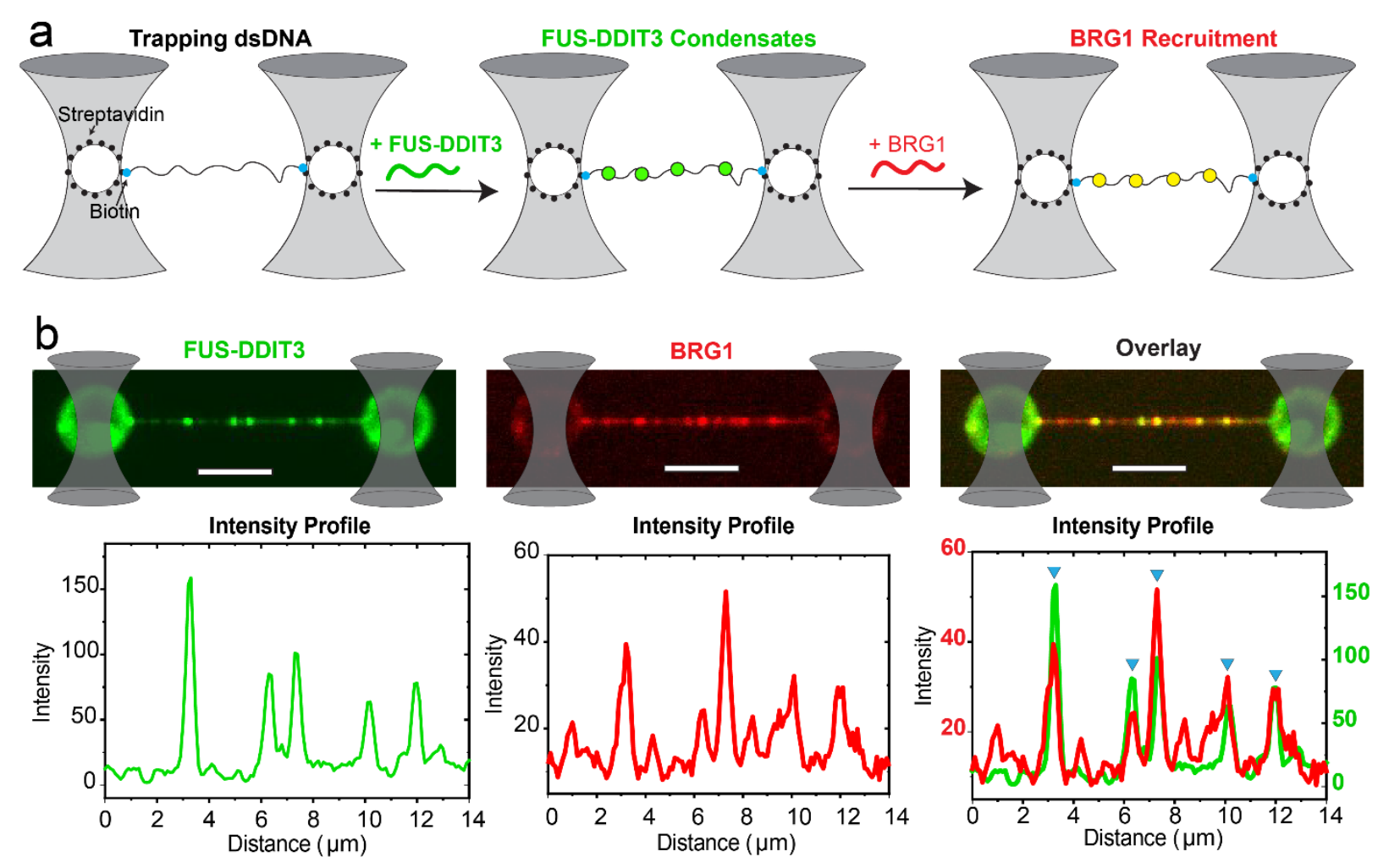

Figure 4. FUS-DDIT3 condensates on dsDNA recruit BRG1. (a) A schematic representation of the single-molecule DNA tethering assay. A single molecule of dsDNA (with ends biotinylated; represented by solid cyan circles) is tethered between two optically trapped polystyrene beads (traps are shown as grey cones; beads are shown as white circles) coated with streptavidin (solid black circles). The transfer of DNA molecule into a microfluidic channel containing FUS-DDIT3 leads to the formation of FUS-DDIT3 condensates (solid green circles). Subsequent transfer of the DNA chain decorated with FUS-DDIT3 condensates to a separate microfluidic channel containing BRG1 leads to the recruitment of BRG1 into the FUS-DDIT3 condensates (solid yellow circles). (b) Experimental data: multicolor confocal fluorescence micrographs and intensity profiles showing the formation of FUS-DDIT3 puncta/condensates (green) on a single DNA molecule followed by the recruitment of BRG1 (red) into the FUSDDIT3 condensates. Blue triangles show the position of the overlapping intensity peaks of FUSDDIT3 and BRG1 representing the recruitment of BRG1 into FUS-DDIT3 puncta/condensates. [FUS-DDIT3] $=250 \mathrm{nM}$ and $[\mathrm{BRG1}]=10 \mathrm{nM}$. The scale bar is $5 \mu \mathrm{m}$ for all images. 


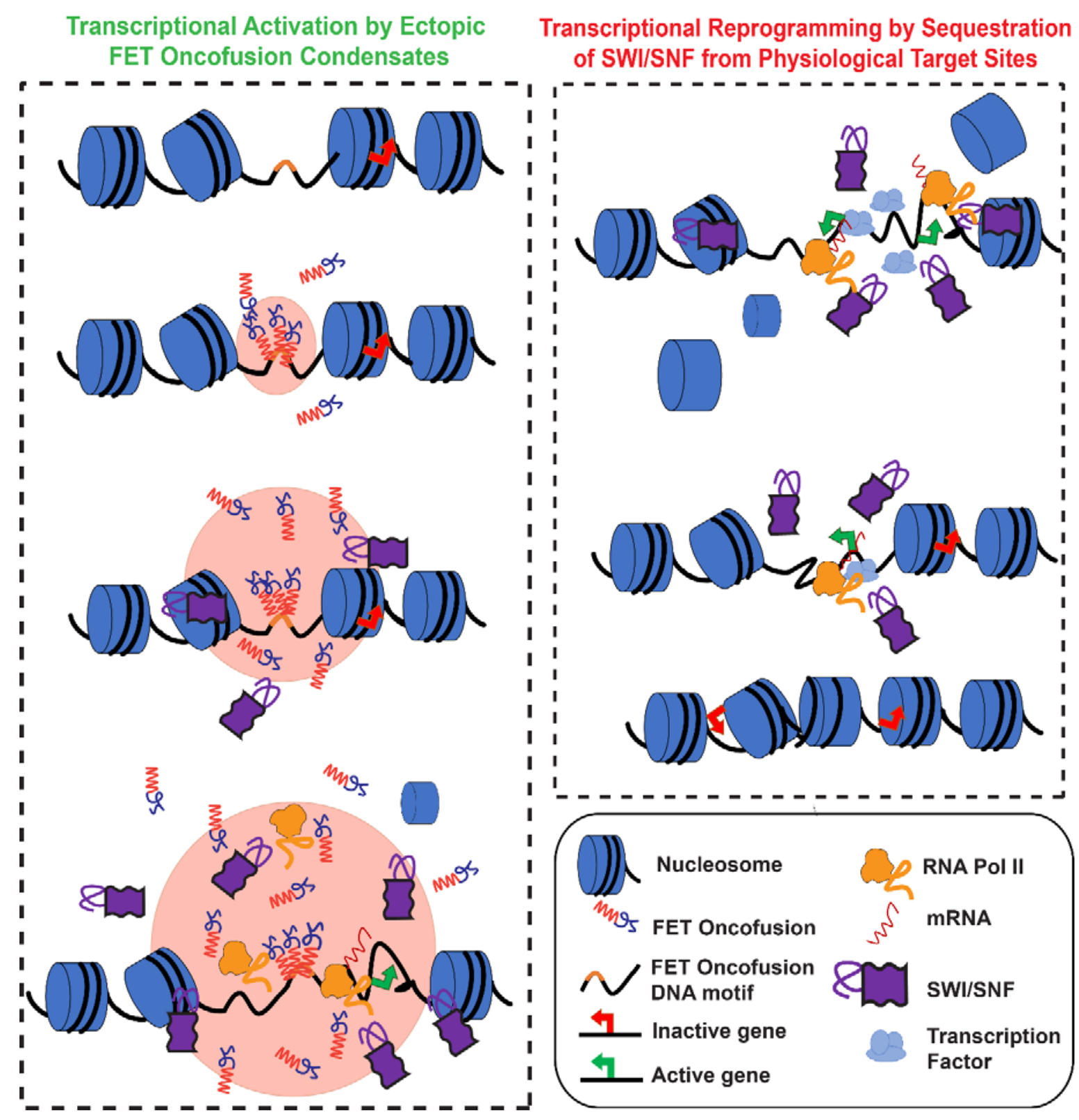

Figure 5. Proposed model for transcriptional reprogramming by FET-fusion oncoproteins. Left: FET fusion proteins can bind to specific DNA motifs defined by the DNA-binding domain at physiologically inactive genes to form phase-separated condensates. The process of LLPS is mediated by the prion-like domain. FET fusion condensates can recruit the ATP-dependent chromatin remodeler SWI/SNF leading to remodeling of the closed chromatin to an open chromatin state, which subsequently provides access to transcriptional coactivators and RNA polymerase II to mediate transcription of otherwise silenced genes. Right: The sequestration of chromatin remodeler SWI/SNF into FUS-DDIT3 condensates, as shown in the left panel, can lead to diminished chromatin remodeling activity at physiologically active genes where continuous SWI/SNF activity is required. This sequestration of SWI/SNF away from the 
bioRxiv preprint doi: https://doi.org/10.1101/2021.04.23.440992; this version posted April 23, 2021. The copyright holder for this preprint (which was not certified by peer review) is the author/funder. All rights reserved. No reuse allowed without permission.

physiological target genes may trigger a switch to a closed chromatin state, thereby decreasing their transcriptional output. 
Table 1: Recurrent fusions of transcriptional regulators with prion-like domains. Legends: DBD - DNA-binding domain; PLD Prion-like domain; PPI motif - Protein-protein interaction motif.

\begin{tabular}{|c|c|c|c|c|c|c|}
\hline $\begin{array}{c}\text { Fusion } \\
\text { Pair }\end{array}$ & $\begin{array}{l}\text { Head } \\
\text { Gene }\end{array}$ & $\begin{array}{c}\text { Head } \\
\text { Junction }\end{array}$ & $\begin{array}{l}\text { Tail } \\
\text { Gene }\end{array}$ & $\begin{array}{c}\text { Tail } \\
\text { Junction }\end{array}$ & Domain Organization & $\begin{array}{c}\text { Implicated in } \\
\text { chromatin } \\
\text { reorganization }\end{array}$ \\
\hline & \multicolumn{2}{|c|}{ Head Gene } & \multicolumn{2}{|c|}{ Tail Gene } & \begin{tabular}{|l|l|l|} 
DBD & PLD & PPI motif \\
\end{tabular} & \\
\hline \multicolumn{6}{|c|}{ (FET protein) - (transcription factor) oncofusions } & Yes $[69,70]$ \\
\hline $\begin{array}{l}\text { FUS- } \\
\text { ERG }\end{array}$ & FUS & 31198157 & ERG & 39755845 & 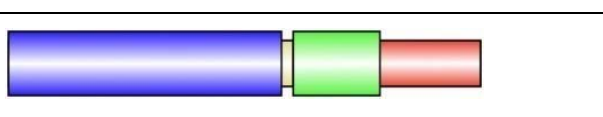 & \\
\hline $\begin{array}{l}\text { FUS- } \\
\text { ERG }\end{array}$ & FUS & 31198157 & ERG & 39763637 & 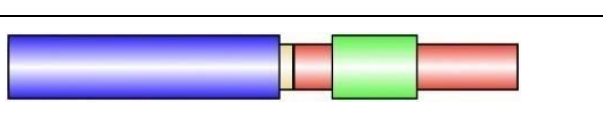 & \\
\hline $\begin{array}{l}\text { EWSR1 } \\
\text {-ATF1 }\end{array}$ & EWSR1 & 29683123 & ATF1 & 51207793 & ] & \\
\hline $\begin{array}{l}\text { EWSR1 } \\
\text {-ATF1 }\end{array}$ & EWSR1 & 29683123 & ATF1 & 51208063 & & \\
\hline $\begin{array}{l}\text { EWSR1 } \\
\text {-ATF1 }\end{array}$ & EWSR1 & 29684775 & ATF1 & 51203238 & & \\
\hline $\begin{array}{c}\text { EWSR1 } \\
\text {-FLI1 }\end{array}$ & EWSR1 & 29683123 & FLI1 & 128675261 & 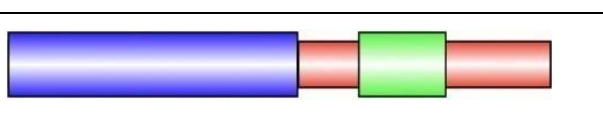 & \\
\hline $\begin{array}{c}\text { EWSR1 } \\
\text {-FLI1 }\end{array}$ & EWSR1 & 29683123 & FLI1 & 128679052 & 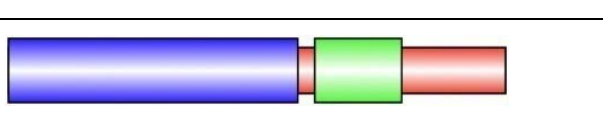 & \\
\hline $\begin{array}{l}\text { EWSR1 } \\
\text {-FLI1 }\end{array}$ & EWSR1 & 29683125 & FLI1 & 128651853 & ${ }^{2}$ & \\
\hline
\end{tabular}




\begin{tabular}{|c|c|c|c|c|c|c|}
\hline $\begin{array}{l}\text { EWSR1 } \\
\text {-NR4A3 }\end{array}$ & EWSR1 & 29688595 & NR4A3 & 102591275 & $2 \sqrt{2}$ & \\
\hline $\begin{array}{l}\text { TAF15- } \\
\text { NR4A3 }\end{array}$ & TAF15 & 34149837 & NR4A3 & 102590321 & J & \\
\hline \multicolumn{6}{|c|}{ (Nuclear receptor) - (transcription factor) oncofusion } & N/A \\
\hline $\begin{array}{l}\text { HEY1- } \\
\text { NCOA2 }\end{array}$ & HEY1 & 80678885 & NCOA2 & 71057083 & -2 & \\
\hline \multicolumn{6}{|c|}{ Fusion between transcription factors } & Yes $[71,72]$ \\
\hline $\begin{array}{l}\text { PAX3- } \\
\text { FOXO1 }\end{array}$ & PAX3 & $\begin{array}{c}22308485 \\
8\end{array}$ & FOXO1 & 41134997 & $\square$ & \\
\hline $\begin{array}{l}\text { PAX7- } \\
\text { FOXO1 }\end{array}$ & PAX7 & 19029790 & FOXO1 & 41134997 & $\square$ & \\
\hline \multicolumn{6}{|c|}{ Fusion between transcription co-factors } & Yes $[67,73]$ \\
\hline $\begin{array}{l}\text { SS18- } \\
\text { SSX1 }\end{array}$ & SS18 & 23615029 & SSX1 & 48123278 & $\square$ & \\
\hline $\begin{array}{l}\text { SS18- } \\
\text { SSX1 }\end{array}$ & SS18 & 23612362 & SSX1 & 48123216 & $\square$ & \\
\hline $\begin{array}{l}\text { SS18- } \\
\text { SSX2 }\end{array}$ & SS18 & 23612362 & SSX2 & 52729628 & $\square$ & \\
\hline
\end{tabular}

